$\xi=\square$

\title{
Philosophy, physics and mathematics of relative and absolute motion
}

\author{
Allan Zade * \\ Independent Researcher \\ *Corresponding author E-mail: AllanZadeUK@gmail.com
}

\begin{abstract}
The idea of motion includes propagation of signals in a given medium and motion of a physical bodies (including observers) relative to the same medium. Combinations of various types of motion lead to many phenomena detectable and hidden from the observer's point of view.

Usually, light offers the most mysterious way of motion regarding the observer. To reveal the truth and eliminate the mystery, we need to use another sophisticated way of imagination and experimentation to reach a clear view on the fundamental processes of motion despite all illusions, old ideas and wrong postulates.
\end{abstract}

Keywords: One-Way Experiment; Space; Duration; Relativity; Signal; Motion.

\section{Introduction}

Motion, velocity, speed and other notions are well known today. In the popular imagination, motion appears as some changes in mutual location of a body relative other bodies (objects). Looking deeper into that process an observer detects some self-controversy in any motion because it appears and disappears regarding the observer's point of view. As soon as the observer changes his/her point of view, motion changes also dramatically.

In any case, when an observer moves with a celestial body observer-to-body relative motion disappears entirely and motion of a body associated with the observer regarding other bodies becomes detectable only by optical observations. The same problem appeared many centuries ago. As a result, an Earth-bound observer associates his motion and a reference frame only with the planet. An observer usually detects motion of the planet in space regarding other celestial bodies by optical observations.

Later research offered many technical devices aimed to physical detection of planetary motion in space regardless of optical observations. Application of those devices became a great challenge to the human mind because it faced motion in some hidden reference frame that was not used ever before. Success and failure of that task depend on the human imagination of fundamental aspects of motion and various methods of velocity detection and determination.

\section{Opposite speed case}

There is an old problem in physics and mathematics about relative motion, relocation and distance covered by a moving body. There are some models that describe relative motion. All of them use a reference frame $(\mathrm{RF})$ to detect and describe motion in that reference frame.

Detection and description of motion in a given reference frame makes no problem for physics and mathematics. By basics defini- tion of motion, velocity $\mathrm{V}$ has the following relationship with distance S (Spatial relocation) and duration ' $D$ ' that the body spends to cover a given distance $\mathrm{S}$.

$\mathrm{V}=\frac{\mathrm{S}}{\mathrm{D}}$

Suppose now that, there are two bodies $\mathrm{M}$ and $\mathrm{N}$ moving with two different velocities $V_{m}$ and $V_{n}\left(V_{m}>V_{n}\right)$. Suppose also that an observer uses the same reference frame for observation of two bodies ( $\mathrm{M}$ and $\mathrm{N}$ ). The observer locates motionlessly in the reference frame. The following figure shows that case.

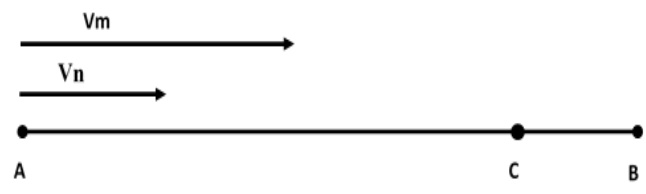

Fig. 1:

Velocities Vm and Vn can be determined only in the same reference frame by the famous equation (1). Therefore, those velocities have the following definitions for the fig. 1 :

$\mathrm{V}_{\mathrm{m}}=\frac{\mathrm{S}_{\mathrm{ab}}}{\mathrm{D}_{\mathrm{m}}}$

$\mathrm{V}_{\mathrm{n}}=\frac{\mathrm{S}_{\mathrm{ab}}}{\mathrm{D}_{\mathrm{n}}}$

In other words, velocity can be determined only by a given distance and some duration that a body (or a thing) spends to cover a given distance. As long as two things arrive at the point of destination (B) simultaneously, both velocities appear equal to each other In case of unequal velocities (in magnitude), a thing "moving faster" reaches the point of destination (B) sooner than another 
thing $(\mathrm{N})$ that uses the lower velocity $\left(\mathrm{V}_{\mathrm{n}}\right)$. In that case, the same duration that causes relocation of the first object (M) by the A-B trajectory, allows the object $\mathrm{N}$ to move only from the point ' $\mathrm{A}$ ' to the point ' $\mathrm{C}$ ' because of a "lesser speed". Therefore, the object ' $\mathrm{N}$ ' needs some extra duration to reach the point ' $\mathrm{B}$ '. That coincides with the equation (1) and shows a general proportion of velocity and duration (greater duration of the process leads to lesser velocity). Everything looks fine until mathematical operations appear in view.

Suppose now that, objects $\mathrm{M}$ and $\mathrm{N}$ move in opposite directions (fig. 2).

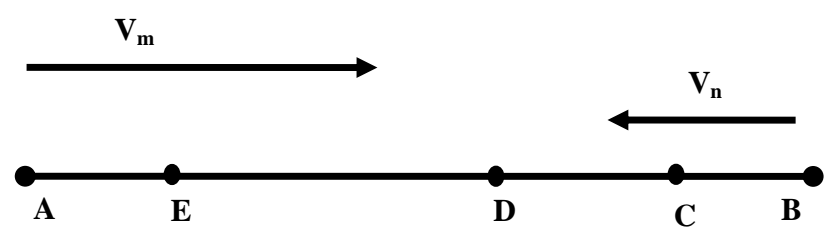

Fig. 2: Opposite Speed Case.

In that case, the general situation has not any change because the object ' $\mathrm{M}$ ' covers the full distance $\mathrm{AB}$ by the same duration $\mathrm{Dm}$ and the second object ' $\mathrm{N}$ ' covers only lesser distance $\mathrm{BE}(\mathrm{AE}=$ $\mathrm{CB}, \mathrm{AC}=\mathrm{BE}$ ). That exactly coincides the first case (fig. 1). However, despite that obvious equality of the first and the second cases, the second experiment (fig. 2) commonly seems like an experiment with the sum of two velocities because two objects move in opposite directions. Possibly, that is an old point of view that reflects the situation when two objects are moving in opposite direction usually hit each other at the end of motion. In that case, the experiment stops immediately at the moment of hit (at the point ' $D$ ').

Despite that obvious way of physical action, two opposite velocities usually appear as some equivalent that summarizes their values.

$\mathrm{V}_{\mathrm{s}}=\mathrm{V}_{\mathrm{m}}+\mathrm{V}_{\mathrm{n}}$

Using equations (2) and (3), the equation (4) can be rewritten the following way

$V_{s}=\frac{S_{a b}}{D_{m}}+\frac{S_{a b}}{D_{n}}=\frac{D_{n} S_{a b}+D_{m} S_{a b}}{D_{m} D_{n}}=\frac{S_{a b}\left(D_{m}+D_{n}\right)}{D_{m} D_{n}}=S_{a b} \frac{\left(D_{m}+D_{n}\right)}{D_{m} D_{n}}$

That equivalent should keep the same law of definition

$\mathrm{V}_{\mathrm{s}}=\frac{\mathrm{S}_{\mathrm{ab}}}{\mathrm{D}_{\mathrm{s}}}$

Therefore,

$V_{s}=\frac{S_{a b}}{D_{s}}=S_{a b} \frac{\left(D_{m}+D_{n}\right)}{D_{m} D_{n}}$

$\frac{1}{D_{s}}=\frac{\left(D_{m}+D_{n}\right)}{D_{m} D_{n}}$

$D_{s}=\frac{D_{m} D_{n}}{\left(D_{m}+D_{n}\right)}$

As a result, there are two different situations. The first situation means motion of two objects in opposite directions. The second situation means motion of the same object with a higher velocity In that case, the full duration of motion of the object with the higher velocity (Vs) between points $\mathrm{A}$ and $\mathrm{B}$ coincides with the duration of opposite motion of two objects (first experiment) from the beginning of the experiment to the moment of meeting of those objects at some point ' $D$ '. Physical process in those two cases looks quite different.

In other words, an observer that determines only duration of both processes detects no difference in motion of the object ' $M$ ' with velocity $\mathrm{Vm}$ between points ' $\mathrm{A}$ ' and ' $\mathrm{D}$ ' (or the object ' $\mathrm{N}$ ' between points ' $\mathrm{B}$ ' and ' $\mathrm{D}$ '), and duration of motion of the image object ' $S$ ' with the velocity Vs between points ' $A$ ' and ' $B$ '. Therefore,

Determination of the duration of the process gives not any information about physical processes of relative motion and cannot help to separate motion of the object in a reference frame and motion of a reference frame relative to the object (Statement A).

\section{Matching speed case}

There is one more "undetectable" aspect in case of subtraction of speeds. Figure 3 shows that case.

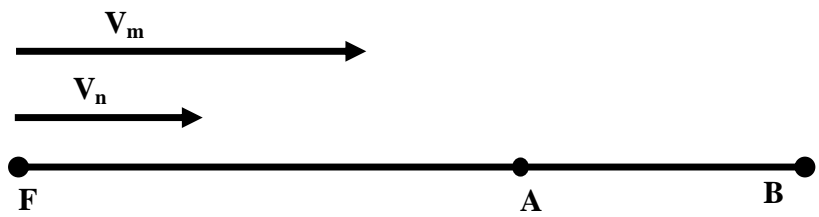

Fig. 3: Matching Speed Case.

Suppose that there are two bodies ' $\mathrm{M}$ ' and ' $\mathrm{N}$ ' moving with different speeds $V_{m}$ and $V_{n}$ in the same direction. In that case, an image body possesses the speed Vs that can be calculated the following way.

$V_{s}=V_{m}-V_{n}$

Using equations (2) and (3), the equation (10) can be rewritten the following way

$V_{s}=\frac{S_{a b}}{D_{m}}-\frac{S_{a b}}{D_{n}}=\frac{D_{n} S_{a b}-D_{m} S_{a b}}{D_{m} D_{n}}=\frac{S_{a b}\left(D_{m}-D_{n}\right)}{D_{m} D_{n}}=S_{a b} \frac{\left(D_{m}-D_{n}\right)}{D_{m} D_{n}}$

That velocity means some value that should be shown by an image body that covers the same distance $\mathrm{AB}$ by the duration that coincides difference of duration that each body spends to cover the same distance. That looks nice for mathematics, but it is bad for physics because such image body does not exist in a physical experiment. Therefore, all speculation about its motion has not any relationship with physical processes of a given physical experiment with given bodies ' $\mathrm{M}$ ' and ' $\mathrm{N}$ '.

In physics, there is another process in matching speed case. Suppose that both bodies mentioned above start their motion from the point ' $\mathrm{F}$ '. The body ' $\mathrm{M}$ ' has velocity $\mathrm{V}_{\mathrm{m}}$ and the body ' $\mathrm{N}$ ' has velocity $\mathrm{V}_{\mathrm{n}}$ as well as in the first experiment. The distance that separates those bodies increases itself in value the more, the greater duration the experiment takes. As a result, the distance separating those bodies has zero value at the beginning of the experiment and rises during the experiment to the full value at the end of the experiment.

The experiment stops as soon as that separating distance reaches the value of AB-distance. In other words, in matching speed case, the body ' $\mathrm{M}$ ' should cover the distance $\mathrm{FB}$ and the body ' $\mathrm{N}$ ' should cover the distance FA (fig. 3). Difference of those distances is equal to the distance $\mathrm{AB}$ that must be covered by the image body ' $\mathrm{S}$ ' for the same duration (!)

Therefore, physical processes in two cases mentioned above look different. In first case, the distance $\mathrm{AB}$ includes paths of two moving bodies $(\mathrm{AB}=\mathrm{AD}+\mathrm{DB}$, fig. 2$)$. In second case, that statement becomes wrong because the path of the body with the greater speed includes the path of the body with the lesser speed and the $\mathrm{AB}$-distance $(\mathrm{FB}=\mathrm{FA}+\mathrm{AB}$, fig. 3). However, mathematical calculations do not show that critical physical difference. That situation leads to the following problem. 


\section{The problem of relative motion}

There is an age-old idea about a determination of relative motion and the observer. They think that the problem can be solved by two signals send in orthogonal direction for two mirrors. Mathematical calculations show this. The duration of the process of forward and backward propagation of the signal depends on the speed of the signal and the observer in a medium. Different orientation of the orthogonal device should show that phenomenon. However, that phenomenon was not ever detected physically. Figure 4 shows that idea in case of fixed location of the observer and figure 5 shows the same idea of measurement in case of motion.

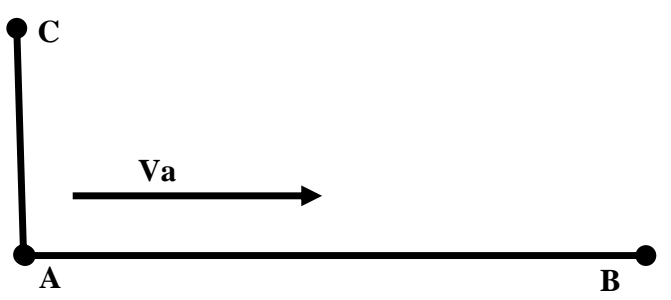

Fig. 4

Figure 4 shows the point ' $A$ ' there the source of the signal is located. The measuring signal moves from the point ' $A$ ' in two orthogonal directions toward points ' $\mathrm{B}$ ' and ' $\mathrm{C}$ '. The signal makes reflection from the mirrors located at those points (B and $\mathrm{C}$ ) and comes back to the point ' $\mathrm{A}$ '. If $\mathrm{AB}=\mathrm{AC}$, then the duration of the signal propagation back and forth in both directions has the same value ever. In that case, $\mathrm{Va}$ (the speed of the observer relative to medium) equals to zero.

As soon as the medium begins motion relative to the observer ( $\mathrm{Va}>$ 0 ) or the observer begins motion relative to the medium the situation changes dramatically. Figure 5 shows that case.

There is a basic distance $A B$ in figure 5. There is an apparatus with two orthogonal arms $\mathrm{AB}$ and $\mathrm{AG}$ (FG). The experiment begins. The signal source emits the measuring signal from the point ' $F$ ' (that coincides with the point ' $A$ ' at the beginning of the experiment). The signal moves in every direction from the point of emission with same speed relative to the medium (Huygens' Principle). The device and the observer move with velocity Va that coincides the direction $\mathrm{AB}(\mathrm{FB})$.

The measuring signal also moves toward point ' $B$ '. However, because of relative motion, the point ' $\mathrm{B}$ ' moves away from the signal. That coincides with the case shown in figure 3 . The only one difference is this. Velocity Vn appears as motion of the reference frame, the device and the observer at the same time.

As a result, the measuring signal spends some extra duration to reach the end of the arm and meets the mirror ' $B$ ' at some point ' $L$ '. Therefore, initial distance of $A B\left(A_{1} B_{1}\right)$ for the signal (in a motionless location of the device with the observer relative to the medium) transforms to some greater distance ' $\mathrm{FL}$ '.

At the next stage, the measuring signal mirrors from the mirror and goes back. The signal meets the point ' $\mathrm{A}$ ' of the apparatus at the point ' $\mathrm{K}$ ' because of motion of the apparatus relative to the medium. At that moment, the arm of the apparatus has location $\mathrm{KB}$ (equal to $\mathrm{A}_{2} \mathrm{~B}_{2}=\mathrm{AB}$ ).

There is a similar situation in case of orthogonal motion of the signal. The signal starts motion from the same point ' $A$ ' of the apparatus that coincides the point ' $F$ ' of the medium at the beginning of the experiment. The signal moves toward some point ' $\mathrm{C}$ ' of the device that coincides with the point ' $G$ ' of the medium. However, unlike the $\mathrm{AB}$ direction, the signal cannot meet the mirror at the point ' $G$ ' because it spends some duration to reach the point ' $G$ '. For the same duration, the point ' $B$ ' of the apparatus moves to the point ' $\mathrm{H}$ ' of the medium and the measuring signal should use path FH instead of FG to meet the mirror.

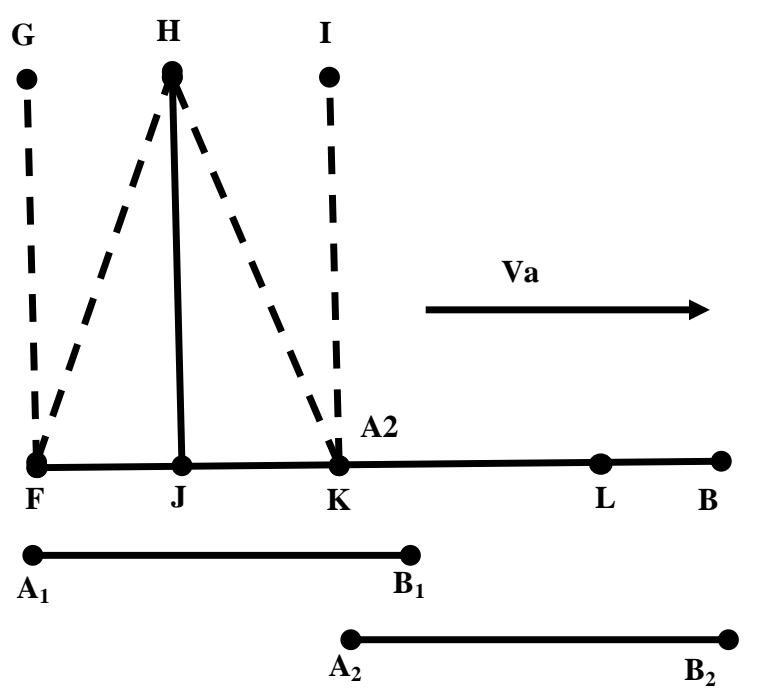

Fig. 5: The Problem of Orthogonal Motion

At the next stage, the signal mirrors at the point ' $\mathrm{H}$ ' and moves back by the path HK. The signal meets the point ' $\mathrm{A}$ ' of the apparatus at the point ' $\mathrm{K}$ ' of the medium.

Distance FHK exceeds distance FGF. Moreover, distance FHK depends on the speed $\mathrm{Va}$ (relative motion of the apparatus and the medium). Therefore, duration of a round-trip signal propagation in $\mathrm{AB}$ direction (that coincides with velocity $\mathrm{Va}$ ) and in the orthogonal direction increases the more the higher is velocity $\mathrm{Va}($ !)

That statement contradicts the common point of view because that point of view uses only projections of the speed Va. Therefore, mathematically, velocity $\mathrm{Va}$ makes no changes in signal propagation in the orthogonal direction. However, it makes significant changes physically.

Therefore,

Physics and mathematics disagree each other in determination of motion in case of linear and orthogonal experiment (Statement B).

\section{The problem of trajectory}

There is one more problem in the mentioned experiment. According to the Huygens' Principle, the measuring signal propagates in every direction from the point of emission and the point of mirroring. Therefore, the detector that moves with the device (associated with the point ' $A$ ') determines full duration only of the signal propagation and cannot determine the exact trajectory of the same signal in a medium-bound reference frame. It creates one more illusion of the observer. The observer thinks that the signal uses some trajectory that coincides with his/her observation. However, a physical path (trajectory) of the measuring signal in a given medium can be different from the observer's imagination.

Strictly speaking, in the orthogonal experiment (observation), the device detects full duration only of the experiment. The following equation shows that mathematically (according to the fig. 5).

$D_{F}=D_{F H}+D_{H K}$

Full duration $\left(\mathrm{D}_{\mathrm{F}}\right)$ has two components $\mathrm{D}_{\mathrm{FH}}$ and $\mathrm{D}_{\mathrm{HK}}$ (of forward and backward propagation of the measuring signal). It looks obvious from the figure 5 that

$D_{F H}=D_{\mathrm{H} K}$

However, it is only a heavy illusion. The device has not any possibility to detect that equality. Therefore, equation (13) becomes only a postulate of the observer.

Figure 6 shows the general case. 


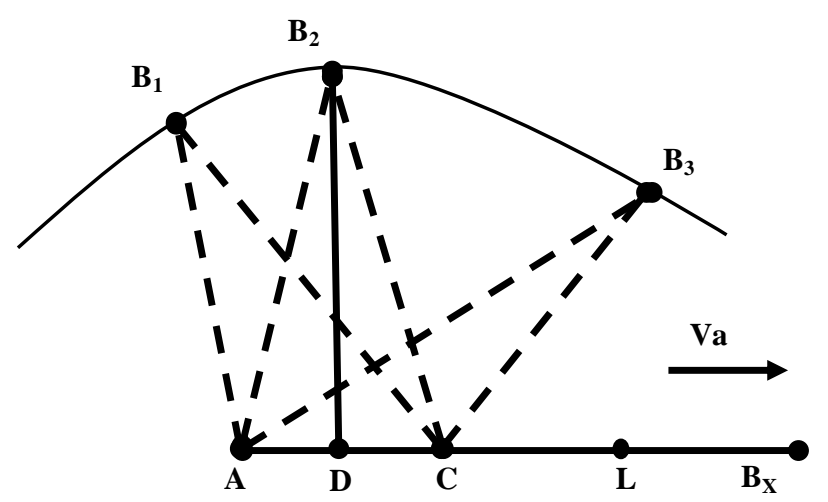

Fig. 6: The General Case of A Signal Trajectory.

Figure 6 represents the same way of relative motion of the apparatus and the measuring signal. The device emits the signal from the point ' $\mathrm{A}$ ' and detects the mirrored signal at the point ' $\mathrm{C}$ '. ' $\mathrm{AC}$ ' is the distance covered by the device during the experiment (during signal propagation).

In general case, the device cannot detect any difference between signal propagation by trajectories $\mathrm{AB}_{1} \mathrm{C}, \mathrm{AB}_{2} \mathrm{C}$ or $\mathrm{AB}_{3} \mathrm{C}$ (or any other $\mathrm{ABnC}$ - trajectory) because the device determines full duration only of the experiment. As a result, full duration ever keeps the same value and follows the same basic law of the signal propagation

$D_{F}=D_{A B n}+D_{B n C}$

However, in general case,

$D_{A B n} \neq D_{B n C}$

That leads to an easy conclusion.

Any device that determines full duration only of a mirrored signal propagation ever shows the same duration of any experiment regardless of a direction of physical motion of the device and medium that supports signal propagation. (Statement $C$ ).

Moreover, 'angular measurement' becomes uncertain that way because initial signal uses one angle with the direction of motion and the mirrored signal uses a different angle. For example, an angle of an initial signal and the direction of motion $\mathrm{B}_{1} \mathrm{AB}_{\mathrm{X}}$ is not equal to the angle of the mirrored signal and the same direction of motion $\mathrm{B}_{1} \mathrm{CB}_{\mathrm{X}}$.

As soon as that angle changes, duration of forward and backward motion of the signal change also but the main equation (14) remains correct. For example, suppose that the initial angle of the signal propagation and the direction of motion becomes $\mathrm{B}_{3} \mathrm{AB}_{\mathrm{X}}$ Obviously, that angle is not equal to angle $\mathrm{B}_{1} \mathrm{AB}_{\mathrm{X}}$. However, the device does not recognize such changes because detectable value (the full duration of the experiment) remains the same (constant).

\section{The geometrical solution}

The explanation given in the previous section remains correct as long as all $\mathrm{Bn}$ points $\left(\mathrm{B}_{1}, \mathrm{~B}_{2}, \mathrm{~B}_{3} \ldots \mathrm{B}_{\mathrm{x}}\right)$ belongs to some curve that keeps the main low of signal propagation (equation 14, statement ' $C$ '). Is it possible to find out that curve? It is certainly possible. There is curve called ellipse that follows the law of equation 14 Equation 15 is also correct for that curve, and equal duration of forward and backward propagation of the measuring signal $\left(\mathrm{D}_{\mathrm{ABn}}\right.$ $\left.=\mathrm{D}_{\mathrm{BnC}}\right)$ becomes only a particular case $\left(\mathrm{B}_{2}\right.$ case $)$ of the experiment.

Ellipse is the locus of points for which the sum of their distances from two fixed points (the foci) is constant. The smaller the distance between the foci, the smaller is the eccentricity and the more closely the ellipse resembles a circle. [1] (Statement D).

A straight line drawn through the foci and extended to the curve in either direction is the major diameter (or major axis) of the ellipse.
Perpendicular to the major axis through the center, at the point on the major axis equidistant from the foci, is the minor axis. [1] (Statement E).

The definition of ellipse gives the following result for signal propagation in the general case (fig. 7).

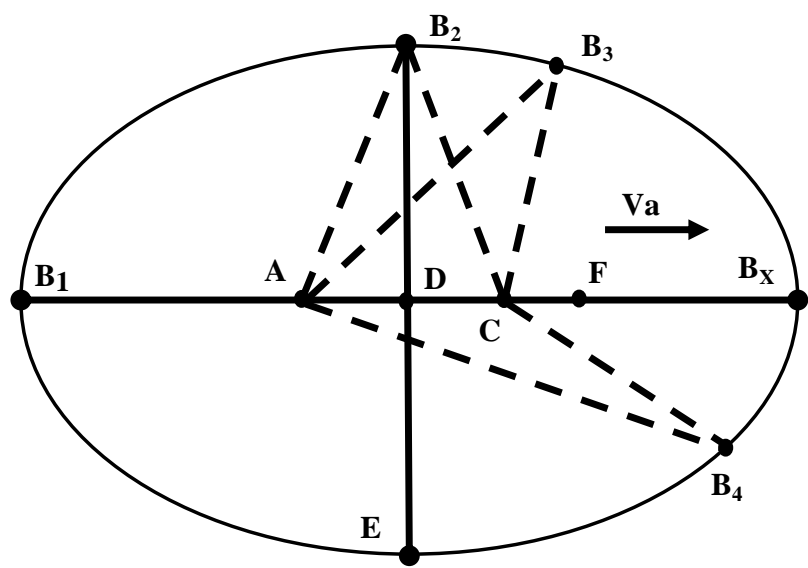

Fig. 7: The Elliptical Law of A Mirrored Signal in A Moving Medium.

Figure 7 shows generalization of the same law of signal propagation mentioned for the figure 6 . In that case, many locations of the point ' $\mathrm{B}$ ' $\left(\mathrm{B}_{1}, \mathrm{~B}_{2}, \mathrm{~B}_{3} \ldots \mathrm{Bn}\right)$ which give the constant duration of full experiment form a perfect ellipse. Points ' $A$ ' and ' $C$ ' become focuses of the ellipse, $B_{1} B_{x}$ becomes the major axis, $\mathrm{B}_{2} \mathrm{E}$ becomes the minor axis of the same ellipse.

In case of motion relative to medium that supports propagation of a measuring signal, a moving transducer detects a constant distance to an object reflecting the measuring signal regardless of direction to the object because full path of the signal toward the object and backward from the object keeps the same constant value as long as the speed of the measuring signal in a medium and the speed of the transducer relative to the same medium remains constant. (Statement $F$ ).

In case of a reference frame bound to a medium that supports propagation of a measuring signal, a moving transducer remains locations in two focuses of an ellipse at the moments of sending and receiving the measuring signal. Location of a body that mirrors the measuring signal and keeps the same distance from the transducer by a duration of a both-way propagation of a measuring signal forms an ellipse that depends on the transducerto-medium uniform relative motion and a given distance between the transducer and the body mirroring the signal. (Statement $G$ ).

Statement ' $G$ ' is the Elliptical Law of a Mirrored signal in a Moving Medium or (ELM) for any measuring signal moving through any medium.

That measurement has one more critical aspect. An observer uses two independent reference frames to make measurements. In a reference frame bound to the observer, the person makes rod-torod measurement by comparison of a unit length (and a given distance) with another "rod" (and a distance that separates the endpoints of a given rod). In that case, all elements of measurement (including the observer) remain in the same reference frame.

In case of a measuring signal, there are two possible situations. As long as a medium remains immovable relative to the observer, all measurements remain equal to rod-to-rod measurement and the measuring signal indeed spends the same duration moving back and forth along a given rod (a unit length). Therefore, the speed of the signal can be found easily by a well-known equation (1).

In case of a moving medium, the situation changes dramatically. The angle between the mirroring body and the direction of motion of the device relative to the medium becomes variable. Despite of that change, a device that makes measurement of full duration only of a measuring signal propagation (or a round-trip experiment) does not "see" (detect, determine, etc.) any changes in data coming from the experiment and a round-trip experiment ever gives the same constant value of signal propagation "along a given 
distance" because of the Elliptical Law of a Mirrored signal in a Moving Medium (ELM). The device cannot recognize such condition because experimental data (duration of a round-trip experiments) are not sufficient to reach a solution of the task and determine the right device-to-medium direction of motion or any change in that direction.

\section{The core problem of comprehension}

The Elliptical Law of a Mirrored signal in a Moving Medium, mentioned above, were formulated regardless any specific signal or medium that supports propagation of a signal. Therefore, that law is applicable to any signal-medium combination without any exception. Here appears an age-old problem.

Michelson-Morley experiment was an attempt to detect the velocity of the Earth with respect to the hypothetical luminiferous ether, a medium in space proposed to carry light waves. First performed in Berlin in 1881 by the physicist A.A. Michelson, the test was later refined in 1887 by Michelson and E.W. Morley in the United States.

The procedure depended on a Michelson interferometer, a sensitive optical device that compares the optical path lengths for light moving in two mutually perpendicular directions. It was reasoned that, if the speed of light were constant with respect to the proposed ether through which the Earth was moving, that motion could be detected by comparing the speed of light in the direction of the Earth's motion and the speed of light at right angles to the Earth's motion. No difference was found. This null result seriously discredited the ether theories and ultimately led to the proposal by Albert Einstein in 1905 that the speed of light is a universal constant. [1]

To answer that question, Michelson and Morley conducted their famous experiment to find the speed of observer-to-light relative motion. They used a specific mathematical model (well-known today) that uses sum and difference of speeds of an observer and the measuring signal. Section 2 of this paper explains a physical difference of mathematical operations with velocities and physical consequences of such operations. Moreover, they used a notion of so-called "Time" to make some calculations and transformation of physical values of velocities. All those calculations are well known today.

The result of their calculations shows this, in case of observer-tomedium relative motion propagation of light in different directions, gives different values of duration of light propagation in a roundtrip experiment (or some anisotropy of light propagation). Using that mathematical model and calculations, they have built a device (well known today as Michelson interferometer) that should confirm their calculations and mathematical model.

To their surprise, the device gives so-called "null" result. In other words, a physical device shows clearly this. The mathematical model, applied to the exponent does not work, and that problem is only a man-made one that appears as the inconsistency of the model and a physical experiment. Despite that obvious result, they insist that the model is correct and made not any attempt to find out a source of their theoretical mistake. Later, the same mathematical model was put on theoretical basis (framework) of a new theory called later 'Relativity'.

They have made one more critical mistake. They have never used their theoretical framework to do experiments with other signals in another medium like acoustical signals in air or water. They were so sure that the model is correct that they paid not any attention to other signal-medium combinations.

However, many decades later, the same experiment with an acoustical signal in the air was conducted with the same "unexplainable" result. It was "Acoustic Michelson-Morley experiment' [2]. That experiment confirms exactly the Elliptical Law of a Mirrored signal in a Moving Medium in case of an acoustical signal in air and stays in contrary to well-known point of view that only light signals show isotropy of propagation in any direction despite motion of the signal source through the medium (instead of theoretically predicted anisotropy). Only ELM explains the result in both cases and supports its applicability to any signal-medium combination.

\section{Beyond the comprehension horizon}

There is one more question about a physical possibility of detection of observer-to-medium relative motion. The question has not any answer in case of a round-trip experiment as shown above. However, the same Elliptical Law of a Mirrored signal in a Moving Medium gives the answer on that question. Figure 7 shows the answer graphically. According the figure, the full length of signal trajectory and duration of signal propagation by that trajectory in case of moving source of a signal remains constant ever $(\mathrm{ABnC}=$ constant).

Despite that problem, a one-way distance between the signal source and the mirroring body changes according to their mutual location and $\mathrm{AB}_{2} \neq \mathrm{AB}_{3} \neq \mathrm{B}_{2} \mathrm{C} \neq \mathrm{B}_{3} \mathrm{C}$, etc. Therefore, to answer the central question about observer-to-signal relative motion, the observer should determine the only one-way duration of signal propagation between a signal source and a mirroring body. Sum of those duration remains constant but individual one-way elements are unique in case of changing mutual location of a signal source and a mirroring body or angle between the direction to a mirroring body and the direction to observer-to-medium (device-to-medium) motion.

All "classical" experiments of signal propagation (including experiments with light) use only a round-trip way of experimentation because they send a signal to a reflector and determine a full duration of a signal that moves back and forth between a signal source and a "mirror". That method guarantees the constant value of duration of a round-trip experiment in any case of constant uniform motion of observer-to-medium and signal-to-medium (because of the Elliptical Law of a Mirrored signal in a Moving Medium). As a result, modern definition of a unit of length has a description by the speed of light.

Any measurement of velocity requires, however, a definition of the measure of length and of time. Current techniques allow a determination of the velocity of electromagnetic radiation to a substantially higher degree of precision than permitted by the unit of length that scientists had applied earlier. In 1983 the value of the speed of light was fixed at exactly $299,792,458$ meters per second, and this value was adopted as a new standard. As a consequence, the meter was redefined as the length of the path traveled by light in a vacuum over a time interval of $1 / 299,792,458$ of a second. Furthermore, the second-the international unit of time - has been based on the frequency of electromagnetic radiation emitted by a cesium-133 atom. [1]

That happens because every Earth-bound laboratory follows the same Elliptical Law of a Mirrored signal in a Moving Medium and detects the same duration of a round-trip experiment in every direction. Therefore,

An observer should use only data of one-way experiments to detect observer-to-medium relative motion in case of experiments with light. (Statement $H$ ).

The statement ' $\mathrm{H}$ ' requires determination of duration at least by two "clocks" located remotely. An indication of clocks depends on internal recurrent physical processes happen in the clocks (like electromagnetic radiation emitting by a cesium-133 atom, as mentioned above) and method of their initial synchronization. The most significant problem here is clock synchronization because one clock ever uses only its indication and the problem does not appear that way (in case of a round-trip experiment). In case of a one-way experiment, two clocks should use some way of synchronization. The result of all later measurements completely depends on that way of synchronization.

There are two major ways of synchronization. Those are direct synchronization and signal-based synchronization. A direct synchronization is independent of any signal propagation in any me- 
dium and ever shows the same indication of two clocks regardless of their mutual location and distance that separate the clocks. The easiest way of direct synchronization is this. An observer puts two "clocks" together and makes synchronization of them. Synchronization means a procedure to set an indication of the second "clock" equal to the indication of the first "clock". A zero distance between clocks at the moment of synchronization guarantees the same indication of both clocks because a signal spends zero duration to reach the second "clock." Later, separated clocks keep the same indication regardless of their mutual location and distance separating them because only their internal recurrent physical processes change indication of each "clock". That exactly matches the idea of direct synchronization.

A signal-based synchronization uses another idea and some postulates. We have not defined a common "time" for A and B, for the latter cannot be defined at all unless we establish by definition that the "time" required by light to travel from A to B equals the "time" it requires to travel from B to A. [5].

In modern times, mathematicians have often used the words postulate and axiom as synonyms. Some recommend that the term axiom be reserved for the axioms of logic and postulate for those assumptions or first principles beyond the principles of logic by which a particular mathematical discipline is defined. [1]

Any way of thoughts that uses something "beyond the principles of logic" often leads to many illusions because the human mind cannot guarantee consistency of logical operations in any area that stays "beyond the principles of logic". (Statement I).

As a result, a mathematician tries to use mathematics everywhere as soon as a person detects something "beyond the principles of logic". That method can be used only in pure mathematics because any other branch of science gives a possibility to check (and eliminate) any illusion created by pure mathematics. That is especially correct for physics because an experiment in physics has not any relationship with a human point of view on the same experiment. Therefore,

A "clock" in a physical experiment should use only the direct synchronization method to avoid any impact from a man-made idea of "right and wrong indication of a clock". (Statement J). In case of a one-way experiment (that was unreachable physically at Einstein's time), the postulate mentioned above becomes only a particular case of ELM. It remains correct only for the orthogonal propagation of a signal regarding observer-to-medium motion. The same statement (postulate) is incorrect in a general case (fig. 7).

There is also a mixed way of clock synchronization that leads to the same result as of direct synchronization method. That method was used in a famous 'CERN faster than light neutrino experiment'.

\section{9. "Faster than light" neutrino experiment}

The OPERA neutrino experiment at the underground Gran Sasso Laboratory has measured the velocity of neutrinos from the CERN CNGS beam over a baseline of about $730 \mathrm{~km}$ with much higher accuracy than previous studies conducted with accelerator neutrinos. The measurement is based on high-statistics data taken by OPERA in the years 2009, 2010 and 2011 [3]

According to the same document, they used a mixed way of clock synchronization. Figure 8 shows that way graphically.

There is a GPS-satellite ' $\mathrm{S}$ ' (fig. 8) that sends a GPS-signal to the start (A) and the end point (B) of the experiment. Each point receives the satellite signal and determines its mutual location regarding the satellite. The determination also gives the distance that separates the point on the Earth surface and the satellite located above the planetary surface. Both points also have "time signal" of the satellite and determine "clock indication" of the satellite.

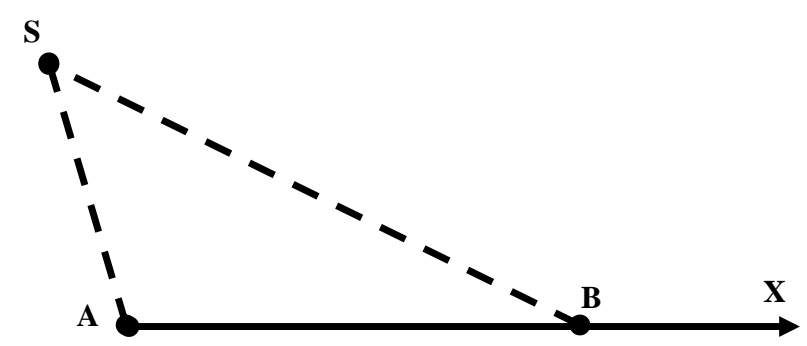

Fig. 8: A Mixed Way of Clock Synchronization by A Satellite.

By that information, both points calculate the duration of a physical signal that GPS-signal spends to cover different distances of SA and SB. After that, both points recalculate indications of their local clocks (or make "synchronization") by the following way.

$T_{A}=T_{N}=T_{S}-T_{S A}=T_{S}-S A / C$

$T_{B}=T_{N}=T_{S}-T_{S B}=T_{S}-S B / C$

where $\mathrm{T}_{\mathrm{N}}$ is some abstract time (or some indication of a "clock") equal to indication of a clock onboard of a GPS-satellite $\left(T_{S}\right), T_{S A}$ and $\mathrm{T}_{\mathrm{SB}}$ are duration of signal propagation from the satellite ' $\mathrm{S}$ ' to point $\mathrm{A}$ or $\mathrm{B}$. Those values were calculated by dividing the distance separating the satellite and a given point (A or B) to the speed of the signal $\mathrm{C}$.

As a result, they recalculate (and set) indication of local clocks at both points by the same indication of a satellite signal $\mathrm{T}_{\mathrm{S}}$ using information of satellite location and distance from each point to the satellite. The result of calculations appears at both points as an indication of satellite clock regardless of the distance that the signal spends to reach different points. In other words, indication $T_{S}$ appears at both points regardless of their mutual location and duration of signal propagation between them and between a satellite and a given point. That result coincides with a direct synchronization method.

Physically, it looks like an indication of a satellite clock appears at both points of Earth-bound receivers simultaneously regardless of any delay caused by signal propagation. As mentioned above, that is a critical condition leading to a correct one-way experiment.

The experiment (by itself) was a one-way experiment because a neutrino beam moves only in one direction and newer comes back again. It is a perfect condition for a one-way experiment. As a result, they fulfill all critical requirements to conduct a one-way experiment. To their surprise, the experiment has shown "unusual" result. From the one hand, it was an unpredictable result at least. From the other hand, there was not any theoretical physicist from the team who recognized a new way of experimentation that exceeds the limitation of Einstein's method of clock synchronization by a light beam (or electromagnetic signal) mentioned above.

According to the documented data about the experiment, they had $730 \mathrm{~km}$ CNGS baseline with a precision of $20 \mathrm{~cm}(731278.0 \pm 0.2)$ m. [3]

The result of the blind analysis shows an earlier arrival time of the neutrino with respect to the one computed by assuming the speed of light $\delta \mathrm{t}$ (blind) $=$ TOFc $-\mathrm{TOF} v=(1048.5 \pm 6.9$ (stat. $)) \mathrm{ns}$. As a check, the same analysis was repeated considering only internal events. The result is $\delta$ t (blind) $=(1047.4 \pm 11.2$ (stat.) $) \mathrm{ns,}$ compatible with the systematic error of $2 \mathrm{~ns}$ due to the inclusion of external events. [3] (TOF is Time Of Flight).

The speed of light in vacuum obtained by all round-trip experiments appears to the observer as C $=299792458 \mathrm{~m} / \mathrm{s}$. Therefore, full duration of a one-way experiment that supposes equality of observer-to-light speed of signal and signal-to-space speed of the same signal gives the following value (duration of the blind experiment).

$D_{B}=S / C=731,278.0 / 299,792,458=0.002,439,280 \mathrm{sec}$ 
The same value of duration is mentioned in the same source [3]. In case of a one-way experiment, they should use geometrical model according to fig. 7 instead of linear one. That experiment coincides with trajectory $\mathrm{B}_{3} \mathrm{C}$ where $\mathrm{CERN}$ locates at the point ' $\mathrm{B}_{3}$ ', and the detector locates at the point ' $\mathrm{C}$ '. As a result, the neutrino beam has some component velocity that moves the detector toward CERN (or toward the point of emission in the space-bound reference frame). Therefore, the duration of the one-way experiment shows some "unusual" result because of SpaceVelocity Va (or observer-to-space relative motion, Absolute Velocity of the observer, etc.). Magnitude and direction of that velocity were unknown at the time of the experiment and experimenters were unable to explain that "unusual" result.

Figure 7 shows clearly this. Vector Va cannot be measured by such experiment because a one-way experiment with a casual orientation of the measuring beam (or another signal) has some unknown angle between the line of the experiment and the direction of the vector. However, the experiment can detect and determine the projection of the vector on the line that was used to do the experiment $\left(\mathrm{B}_{3} \mathrm{C}\right)$. That is the straight line between CERN and the detector. That component speed can be calculated easily according to figure 2 (sum of velocities).

Full duration of the experiment $\left(D_{L}\right)$ becomes lesser than expected

$D_{L}=0.002,439,280 \mathrm{sec}-0.000,001,048,5 \mathrm{sec}$

$$
=0.002,438,231,5 \mathrm{sec}
$$

That component speed excludes some duration from the experiment by reducing the physical length of neutrino path in space (or in the space-bound reference frame). That value can be found the following way.

\section{$\mathrm{Sn}=(\mathrm{TOFc}-\mathrm{TOFv}) \cdot \mathrm{C}=0.000,001,048,5 \mathrm{sec} \times 299,792,458 \mathrm{me}-$} ters $/ \mathrm{sec}=314.332$ meters

Therefore, the component speed $\left(\mathrm{V}_{\mathrm{S}}\right)$ moves the detector toward the point of emission (CERN) for Sn meters by the duration $D_{L}$ Those values can be used to determine the magnitude of that component speed by the easiest way.

$$
\begin{aligned}
& V_{S}=S n / D_{L}=314.332 / 0.002,438,231,5=128,918 \mathrm{~m} / \mathrm{s} \\
& =129 \mathrm{~km} / \mathrm{sec}
\end{aligned}
$$

The component speed $\left(\mathrm{V}_{\mathrm{S}}\right)$ appears only as some part of the full speed of observer-to-space motion (or Earth-to-space motion, Earth-to-ether motion, etc., in that particular experiment). It exceeds the speed of orbital motion of the Erath many times and becomes closer to the orbital speed of the solar system in the galaxy. The full velocity of Earth-to-space motion should be greater and should exceed an optically obtained speed of the Solar system in the galaxy. That extra speed should show relative motion of the galaxy core to space.

It is also possible to calculate a ratio of signal-to-observer motion regarding the medium (space). By the same data, the ratio obtained by duration $\left(R_{D}\right)$ and the ratio obtained by speed $\left(R_{S}\right)$ take the following values.

$$
\begin{aligned}
& \mathrm{R}_{\mathrm{D}}=\mathrm{D}_{\mathrm{L}} /(\mathrm{TOF}-\mathrm{TOF} v)=0.002,438,231,5 / 0.000,001,048,5 \\
& =2,325.45 \\
& \mathrm{R}_{\mathrm{S}}=\mathrm{C} / \mathrm{V}_{\mathrm{S}}=299792458 / 128,918=2,325.45 \\
& \mathrm{R}_{\mathrm{D}}=\mathrm{R}_{\mathrm{S}}
\end{aligned}
$$

Equation (24) shows a mathematical interpretation of fig. 2 because the ratio of velocities and ratio of the duration of each part of the experiment are ever equal to each other.
The explained experiment had some instrumental error caused by propagation of a physical signal from the satellite to both points of the Earth surface because of undetectable relative motion of the reference frame containing all elements (the satellite and both points on the Earth surface) and the medium itself (space).

Later investigation of the result caused another claim about the lesser value or complete absence of anything mention in the initial claim and document. That situation raises more questions than answers because the staff that conducted the experiments and measurements was experienced one in a given area of research. The biggest problem with the initial claim was absence of a theory that supports such type of experiments. However, absence of $a$ proper theory means not a necessity of ignoring of a result of physical measurements because only physical experiments can eliminate mathematical (and other) illusions as mentioned above.

\section{Other relative experiments}

There is an old question about Michelson-Morley experiment. Is it a unique one or various signals in other medium show the same result? Surprisingly, that information almost unreachable because data from such experiments are strictly limited (why?). However, a German researcher had conducted Michelson-Morley experiment themselves in another signal-medium combination. That was an acoustic Michelson-Morley experiment. The result was astonishing.

An ultrasonic range finder was mounted on a horizontally rotatable rail at fixed distance, $s$, to a reflector on the top of a car. The change of the distance reading, s, determined the two-way velocity of sound as a function of the car's velocity and direction. As a result of this experiment, the out and back velocity $\mathrm{C}_{2}$ was determined to be isotropic - as in the optical case of the Michelson-Morley experiment. Within the experimental error, the velocity was found to vary as $C_{2}=\left(C^{2}-V^{2}\right) / C$. [2].

That experiment supports exactly the Elliptical Law of a Mirrored signal in a Moving Medium mentioned above. There is also some inconsistency in terminology here. A round-trip experiment obtains full duration only of a signal moving back and forth during the experiment. A one-way experiment obtains a duration from the one-way propagation of a measuring signal. Two-way experiment means two one-way experiments and gives full information from the experiment that shows two values of duration of forward and backward motion of a measuring signal. Therefore, the experiment described by Mr. Feist was a round-trip experiment as well as the famous Michelson-Morley experiment with light.

No evidence was found in the literature of an acoustic analogue of the MME with sound waves. After SRT decoupled both fields, developing sound ray sources and experimenting with them would not have produced a stimulus equivalent to those performed with lasers. [2]

The absence of "a stimulus equivalent to those performed with lasers" makes all researchers blind with the idea of "uniqueness" of light and its "reference frame unexplainable regarding other motion". That was the same logical mistake done by $\mathrm{Mr}$. Michelson earlier. Michelson and Morley made a claim about the uniqueness of their optical tests before full-scale tests with other signals in another medium (including acoustic tests) to be sure that their mathematical model gives the right description of all possible experiments.

The mathematical model used by Michelson and Morley was restricted by (and applicable only for) round-trip experiments with light moving through space. (Statement $K$ ).

Despite their illusion, a physical experiment gives correct result immediately. It shows not any anisotropy of light propagation. This outcome is entirely compatible with the Elliptical Law of a Mirrored signal in a Moving Medium and can be used as one more evidence that proves the general law of signal propagation in every medium. Later experiments with acoustic signals show the same law (like Feist's experiments). 
One more evidence in the same area or research comes from experiments conducted by Mr. De Witte. In 1991 Roland De Witte carried out an experiment in Brussels in which variations in the one-way speed of RF waves through a coaxial cable were recorded over 178 days. The data from this experiment shows that De Witte had detected absolute motion of the earth through space, as had six earlier experiments, beginning with the Michelson-Morley experiment of 1887 [4]. (There is some doubt in the given citation about MMX because MMX made not any detection of absolute motion of the Earth through space.)

He used two sets of atomic clocks in two buildings in Brussels separated by $1.5 \mathrm{~km}$ and the research project was an investigation of the task of synchronizing these two clusters of atomic clocks (!) To that end $5 \mathrm{MHz}$ radio frequency (RF) signals were sent in both directions through two buried coaxial cables linking the two clusters. [4]

In that case, propagation of a signal from one cluster (set) of atomic clocks to another one reproduces exactly a one-way experiment because a signal never comes back to the point of initial transmission during the experiment. If they send a signal forward, and backward they had a chance to conduct a two-way experiment that shows a duration of forward and backward propagation of a measuring signal separately. However, they failed because they had not any way of pure direct synchronization of two clusters of atomic clocks. As mentioned above, any experiment with a signal moving back and forth between those clusters is not sufficient for the solution of the task of direct synchronization (explained in details in the next section).

Despite those problems, Mr. De Witte detected some counterarguments to basic postulates of Relativity experimentally. Moreover, he detected exact dependence of his measurements with sidereal days that was not detected in MMX [4].

Is it possible to solve all those puzzles and put all things in their right places? Is it possible to make measurements by another device or method that can confirm or reject "strange" results, claims and some theories coming from some unknown area of physics? Today, there is a definite answer on all those questions There is a patent application WO/2015/040505 describing a unique device called a Signal Medium Motion Measurement Apparatus or SMA and published the last year by World Intellectual Property Organization (WIPO), Geneva, Switzerland.

\section{A signal medium motion measurement apparatus (SMA)}

The apparatus uses the universal approach to measurements. There is a direct synchronization method mentioned above. In case of CERN neutrino experiment direct synchronization were done by detecting the same satellite signal at two distant points and recalculation of indication of clocks by a known distance from a given point to the satellite.

SMA uses another direct synchronization method (pure synchronization method). Moreover, to avoid any relationship with man-made categories, the apparatus uses not any notion regarding "Time" because that category has many internal controversies and should not be used in physical experiments. An apparatus contains only an Oscillating Device (OD) and Counting Device (CD). All measurements appear only as some oscillations done by the OD and counted by CD. A physical realization of both devices has only one restriction. Frequency (and stability) of oscillations should be enough to determine the propagation of a given signal between apparatuses in a given medium.

To make synchronization of CD both apparatuses should be put together and the second apparatus sets an indication of its $\mathrm{CD}$ to the value equal for indication of the $\mathrm{CD}$ of the first apparatus (by a measuring signal). The zero distance between apparatuses at the moment of synchronization guarantees the same indication of both CD. After synchronization, the second apparatus moves away to a given distance and becomes ready to detect a measuring signal That procedure utilizes the Local Synchronization and Remote
Operation Method (LSROM). That method guarantees a clear measurement of a signal propagation from one apparatus to another one without any impact of any other signal propagation and delays caused by any other physical processes. That method is also free of any other human assumption or postulate.

In case of light or other electromagnetic signals, that is a critical aspect of apparatus' operability. For example, we have not defined a common "time" for A and B, for the latter cannot be defined at all unless we establish by definition that the "time" required by light to travel from A to B equals the "time" it requires to travel from B to A. [5] That is only a man-made postulate that was acceptable at 1905 , but modern equipment exceeds the limitation of "clock synchronization" by an electromagnetic signal. LSROM mentioned above goes beyond that limitation as well as all other Direct Synchronization Methods (DSM).

In case of a Direct Synchronization Method, the same indication of a counting devices at remotely located apparatuses appears regardless of any duration that an electromagnetic signal spends to cover the distance that separates those apparatuses. (Statement $L)$.

SMA can be used in any signal-medium combination because it makes measurement of the duration of signal propagation in a given medium from one apparatus to another one. In case of electromagnetic signals, that method means one-way measurement (and experiments) of light propagation. That operability is unreachable for any other device that uses only a round-trip measurement (experiments with a mirrored electromagnetic signal).

A one-way measurement of signal propagation is the only one possible way to separate duration of forward and backward propagation of a signal. (Statement $M$ ).

Therefore, SMA is the only one apparatus known for today that can detect and determine the Elliptical Law of a Mirrored signal in a Moving Medium (ELM) by physical measurements.

\section{The general problem of orthogonal mode}

SMA makes measurements of a one-way experiment or two oneway experiments in "opposite directions". Data coming from those experiments can be use in various mathematical models depending on a model that is better for a given situation (measurment). In case then a signal-to-medium speed of motion is considerably higher than an observer-to-medium speed of motion (SMA-tomedium motion) the observer can use the easiest model of interpretation of data from SMA. In that case, SMA-to-medium relative motion can be determined separately in every direction of measurement by different arms of the device. That easiest mathematical method has shown in the patent application for one and three-arm device (orthogonal device or TSVD device/mode).

However, it is possible to apply another mathematical model to a determination of SMA-to-medium motion and the speed of signalto-medium motion. That is a full mathematical model. Figure 7 shows that the full path of a measuring signal never coincides with the distance seen by a moving observer. For example, the observer sees a straight line $\mathrm{B}_{2} \mathrm{D}$ and thinks that the measuring signal moves along the line. That is correct only in the reference frame bound to the observer. However, because of ELM, the measuring signal uses a different trajectory $\mathrm{AB}_{2} \mathrm{C}$ that depends on the observer-to-medium speed of relative motion. That speed creates some distance between points ' $\mathrm{A}$ ' and ' $\mathrm{C}$ ' that look like the same point ' $D$ ' from the observer's point of view (in the reference frame bound to the observer).

As a result, SMA can use the following complex method in the form of a general equation to determine the speed of the measuring signal in the medium and the speed of its motion relative to the same medium (fig. 7).

$\frac{R_{1}}{C}+\frac{R_{2}}{C}=\frac{R_{S}}{V}$ 
where $\mathrm{C}$ is the speed of the measuring signal relative to medium (from the observer's point of view), $R_{1}$ (Huygens' Radius number one) is the distance covered by the measuring signal going from the point $\mathrm{A}$ to the point $\mathrm{B}, \mathrm{R}_{2}$ (Huygens' Radius number two) is the distance covered by the measuring signal going from the point $\mathrm{B}$ to the point $\mathrm{C}, \mathrm{V}$ is the speed of observer-to-medium relative motion, Rs - is the distance covered by the observer (SMA) during the experiment. There is also value $\mathrm{N}$ as the rate of signal-tomedium and observer-to-medium motion $(\mathrm{N}=\mathrm{C} / \mathrm{V})$. Equation (25) can be rewritten using a definition of $\mathrm{N}$ the following way.

$\frac{R_{1}}{N V}+\frac{R_{2}}{N V}=\frac{R_{S}}{V}$

SMA obtains value $R_{1}$ as $C_{1}\left(D_{1}\right.$ is a duration of forward propagation of a measuring signal), $R_{2}$ as $C D_{2}\left(D_{2}\right.$ is a duration of backward propagation of a measuring signal), $R_{S}$ as $V_{S}\left(D_{S}\right.$ is a full duration of the experiment, $\mathrm{D}_{\mathrm{S}}=\mathrm{D}_{1}+\mathrm{D}_{2}$ ). Therefore,

$\frac{C D_{1}}{N V}+\frac{C D_{2}}{N V}=\frac{V D_{S}}{V}$

$\frac{N V D_{1}}{N V}+\frac{N V D_{2}}{N V}=\frac{V D_{S}}{V}$

$D_{1}+D_{2}=D_{S}$

The basic equation (25) transforms to the form (29) that leaves not any possibility to have a solution any possible way (by mathematics). The same equation is applicable to any arm of SMA. That also leaves not any possibility to reach a solution of the equation and find values $\mathrm{N}$ and $\mathrm{V}$. For example, in case of two arms, the equation takes the following form.

$D_{3}+D_{4}=D_{S}$

where $D_{3}$ and $D_{4}$ are duration of measuring signal propagation back and forth along the other arm of SMA.

However, SMA can be used for determination of all values in a given complex model. SMA needs only to change a mode of operation and use the Radial Mode (RM) of operation instead of orthogonal mode (OM or angular mode).

\section{The radial mode of operation}

The radial mode is the best one when an observer likes to determine precisely correct values of the speed of the measuring signal in the medium and observer-to-medium relative motion despite the physical complexity of the detecting device. Figure 9 shows that mode of operation.

There is a circle ' $\mathrm{O}$ ' $\left(\mathrm{B}_{0} \mathrm{~B}_{1} \mathrm{~B}_{2}\right)$ and a semicircle ' $\mathrm{E}$ ' $\left(\mathrm{B}_{0} \mathrm{BB}_{2}\right)$ in the figure. Planes of those circle and semicircle are perpendicular to each other. The device uses two apparatuses only. One of them is located at the point ' $A$ ' (the center of the circle ' $O$ ' and semicircle ' $E$ '). Another one is movable and can move along the line from the first apparatus to the semicircle ' $E$ '. The semicircle ' $E$ ' is also movable and can rotate by points $\mathrm{B}_{0}$ and $\mathrm{B}_{2}$ sliding the circle ' $\mathrm{O}$ '.

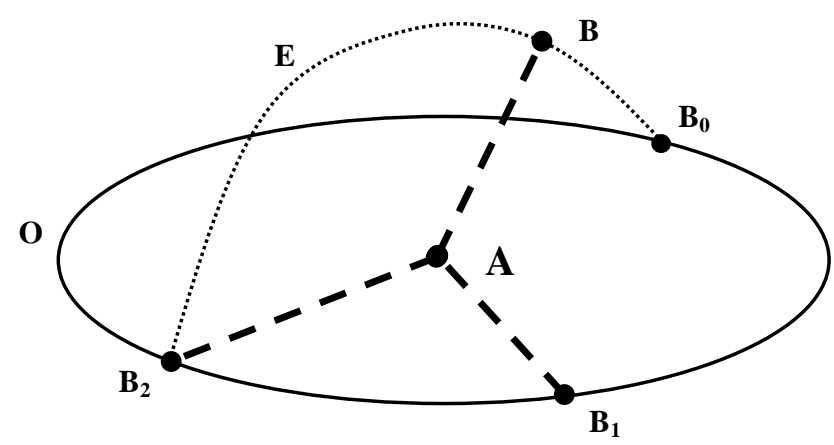

Fig. 9: SMA in the Radial Mode of Operation.
Apparatuses use the same way of synchronization (LSROM) as well as in the orthogonal mode. The measurement has two stages. At the first stage, apparatus ' $\mathrm{B}$ ' takes some location $\mathrm{B}_{1}$. It makes some distance $\mathrm{AB}_{1}$ between both apparatuses. The apparatuses make a measurement of measuring signal propagation in forward and backward direction. Apparatus B begins to move along the circle ' $\mathrm{O}$ ' comparing the duration of forward and backward signal propagation. As soon as that difference reaches the maximal value, the apparatus B stops its motion along the circle ' $O$ '. That means this. Projection of vector of SMA-to-space motion coincides with the straight line connecting both apparatuses in the horizontal plane. That is location $B_{2}$ in figure 9 . Therefore, $A B_{2}$ is the line of projection of the vector and the vector itself exists in the plane of the semicircle ' $\mathrm{E}$ '.

To find that vector, the apparatus B moves along the semicircle ' $E$ '. It compares the duration of signal propagation in forward and backward direction with some steps of location and searches the exact location that gives the maximal difference of those values. That is location B. Therefore, a maximal difference in those values means the measurement of the vector itself instead of any of its projection. Hence, SMA determines a real direction of the vector (SMA-to-space motion). That is a mechanical solution to find the direction of the vector.

To determine a magnitude of the vector, SMA makes easy calculations. It determines two velocities (of forward and backward motion) of the measuring signal $\mathrm{V}_{\mathrm{F}}$ (velocity of forward motion) and $V_{B}$ velocity of backward motion along the straight line connecting the apparatuses (AB-line) by the following way.

$V_{F}=\frac{L}{D_{F}}$

$V_{B}=\frac{L}{D_{B}}$

where, $\mathrm{L}$ is the length of the measurement line ( $\mathrm{AB}$ distance), $\mathrm{D}_{\mathrm{F}}$ is a duration of forward motion of the measuring signal, $D_{B}$ is a duration of backward motion of the measuring signal.

By basic equations of the velocities, there are two elements in each case. Those are the speed of signal-to-medium motion (E, or Electromagnetic Signal Space Speed) and the speed of SMA-tomedium motion (observer-to-medium motion) (V)

$V_{F}=E+V$

$V_{B}=E-V$

Therefore,

$V_{F}=E+V ; \mathrm{V}=V_{F}-E ; V_{B}=E-\left(V_{F}-E\right)=2 E-V_{F} ;$ $2 \mathrm{E}=V_{F}+V_{B}$;

(35)

$\mathrm{E}=\left(V_{F}+V_{B}\right) / 2$

The same way gives the following value of $\mathrm{V}$.

$\mathrm{V}_{\mathrm{B}}=\mathrm{E}-\mathrm{V} ; \mathrm{E}=\mathrm{V}_{\mathrm{B}}+\mathrm{V} ; \mathrm{V}_{\mathrm{F}}=\left(\mathrm{V}_{\mathrm{B}}+\mathrm{V}\right)+\mathrm{V}=2 \mathrm{~V}+\mathrm{V}_{\mathrm{B}} ;$

$2 \mathrm{~V}=\mathrm{V}_{\mathrm{F}}-\mathrm{V}_{\mathrm{B}}$

(37)

$\mathrm{V}=\left(\mathrm{V}_{\mathrm{F}}-\mathrm{V}_{\mathrm{B}}\right) / 2$

Equation (36) shows this. The speed of signal-to-medium motion (E) equals to the speed of observer-to-signal motion (C) only in one case then $V_{F}=V_{B}$. That means equal duration of forward and backward motion of a measuring signal $\left(D_{F}=D_{B}\right)$ in every direction and coincides with the Einstein's postulate mentioned above. However, that is only a particular case. In general case, $D_{F}$ $\neq \mathrm{D}_{\mathrm{B}}$ and $\mathrm{V}_{\mathrm{F}} \neq \mathrm{V}_{\mathrm{B}}$, and the same postulate becomes wrong.

In general case, physical trajectory of a measuring signal does not match the straight line of a physical measurement device that an 
observer uses to make measurements. As a result, the Elliptical Law of a Mirrored signal in a Moving Medium causes longer duration of the experiment and $\mathrm{C}$ (the speed of a measuring signal in the observer-bound reference frame) becomes dependent on $\mathrm{V}$ (the speed of observer-to-medium motion). As a result, E appears ever greater than $\mathrm{C}(\mathrm{E}>\mathrm{C})$. Only the radial mode of operation of SMA exceeds that limitation by preliminary orientation (before the final measurement) of the device that the line connecting the apparatuses coincides the direction of observer-to-medium motion. That is the only one correct method of measurement that helps the observer to apply the unit length from the observer-bound reference frame to the medium-bound reference frame because of the fundamental equation of speed (1).

\section{Conclusion}

Each theory depends on the facts and regularities which were put into considerations at the time of the creation of the theory and some facts and regularities which were rejected [6]. Some theories have basic postulates also. Those postulates remain correct until some technology exceeds the human imagination and "reasonable limitations" put in the theoretical framework of a theory. In that case, application of new technology to an old problem leads to an entirely new point of view. Every major technological invention shows the same impact on theoretical knowledge. The best example of that is work of Galileo Galilei, who revolutionized astronomy when he applied the telescope to the study of extraterrestrial bodies in the early 17th century. [1]. That device eliminated many old illusions and changed the human mind and human imagination of the human role in the universe completely.

SMA makes the same impact. It destroys many basic postulates of 19-th and 20-th century physics and becomes a useful tool to check any postulate of a later-created theory.

For example, LSROM can be used to make synchronization of counting devices of many apparatuses. Four apparatuses are enough to conduct a double-ray experiment. According to the Einstein's point of view, any light beam has the same speed $\mathrm{C}$ measured by any observer regardless of his/her state of motion. Therefore, the same observer takes the same value of $\mathrm{C}$ of a light beam if he/she moves at the speed of light $\mathrm{C}$. Figure 10 shows the double-ray experiment.

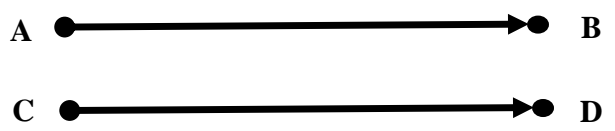

Fig. 10: A Double Ray Experiment.

A Double-Ray Experiment (DRE) uses four apparatuses synchronized by LSROM. Therefore, all counting devices have the same indication during the experiment. The apparatuses located at points A, B, C and D. At a given indication of a counting device, apparatuses $\mathrm{A}$ and $\mathrm{C}$ emit two measuring signals to apparatuses B, and D. Einstein's theory gives not any answer on any question regarding that experiment because each ray should be faster than another one. SMA determines the exact (physical) duration of the experiment and both electromagnetic signals (rays) spend the same duration to cover the same distance. That theoretical prediction can be easily proved physically by a DRE.

Only SMA offers a complete set of experiments to check every postulate of Relativity and any later-created theory regarding propagation of any signal in any medium.

\section{References}

[1] Encyclopedia Britannica Deluxe Edition, Chicago, 2008.

[2] Feist Norbert, „Acoustic Michelson-Morley Experiment with an Ultrasonic Range Finder", proceedings of the NPA, Vol. 6, No. 2, (2010)
[3] Adam T. and others, „Measurement of the neutrino velocity with the OPERA detector in the CNGS beam", (2012) (http://static.arxiv.org/pdf/1109.4897.pdf).

[4] Cahill Reginald T., ,The Roland De Witte 1991 Detection of Absolute Motion and Gravitational Waves", Progress in Physics 3, 60-65, (2006)

[5] Einstein Albert, „On the electrodynamics of moving bodies“, published in a book The Principle of Relativity, Methuen and Company, Ltd., London, 1923.

[6] Zade Allan, „Z-Theory. Foundation and Frame“, Journal of Education and Vocational Research Vol. 5, No. 4, pp. 191-197, (2014) (ISSN 2221-2590). 